\title{
Galactic Centre science with an ELT
}

Thibaut Paumard ${ }^{1,2, a}$, Yann Clénet ${ }^{1,2}$,

Richard Davies ${ }^{3}$, Reinhard Genzel ${ }^{3,4}$, and the MICADO team,

Matthew Lehnert ${ }^{5}$, Jean-Gabriel Cuby ${ }^{6}$, Simon Morris ${ }^{7}$, and the EAGLE team.

${ }^{1}$ Laboratoire d'Études Spatiales et d'Instrumentation en Astrophysique (LESIA) Observatoire de Paris / CNRS / UPMC / Université Paris Diderot

5 Place Jules Janssen, 92190 Meudon, France

2 PHASE (Partenariat haute résolution angulaire sol espace) partnership between ONERA, Observatoire de Paris, CNRS and Université Paris Diderot

3 Max-Planck-Institute for extraterrestrial Physics (MPE), Gissenbachstrasse 1, 85741 Garching, Germany

${ }^{4}$ Department of Physics, Le Conte Hall, University of California, CA 94720 Berkeley, USA

5 Galaxies Étoiles Physique et Instrumentation (GEPI) Observatoire de Paris / CNRS / Université Paris Diderot

5 Place Jules Janssen, 92190 Meudon, France

${ }^{6}$ Laboratoire d'astrophysique de Marseille (LAM)

Observatoire de Haute Provence / CNRS / Université de Provence Aix-Marseille 1, Pôle de l'Étoile Site de Château-Gombert, 38 rue Frédéric Joliot-Curie, 13388 Marseille CEDEX 13, France

7 Durham University, University Office, Old Elvet, Durham DH1 3HP, UK

\begin{abstract}
VLT and the Keck Telescope have allowed tremendous progress on the understanding of environment of $\mathrm{Sgr} \mathrm{A}^{*}$, the supermassive black hole at the Galactic Centre. However, these telescopes and associated instrumentation are reaching limitations which can only be overcome with larger apertures. We will summarise the most recent results in this area: star and gas dynamics, the origin of massive stars in the central parsec, the detection of stars on almost relativistic orbits. We will then anticipate the results that two E-ELT projects, MICADO and EAGLE, are expected to allow.
\end{abstract}

\section{Introduction}

\subsection{The Galactic Centre: a unique laboratory}

It is suspected that most if not all galactic nuclei host a massive black hole, whose mass is tightly correlated with the properties of the galaxy. It seems that the evolution of the black hole and its host galaxy are closely interrelated. Actually, very little is currently understood of the birth and growth of nuclear black holes or, indeed, galaxies.

Although they can reach galactic masses $\left(10^{9}\right.$ solar masses in some cases $)$ these black holes are tiny compared to intergalactic distances, and therefore their study is quite challenging. The closest of the supermassive black-holes is Sgr A*, at the centre of the Milky Way galaxy. The favourable compromise between its distance and intrinsic size make it the largest of all black-hole candidates (in projection).

The Galactic Centre is a unique laboratory to study the processes at work in galactic nuclei, such as co-evolution of supermassive black holes and their host galaxy, nuclear star formation and gravity in the strong field limit. However, it is a complex environment. Current studies are confusion-limited in both the spatial and spectral dimensions. An ELT has the potential to provide the required spatial resolution and sensitivity to overcome these limitations.

\footnotetext{
a e-mail: thibaut.paumard@obspm.fr
} the original work is properly cited. 
We summarise here the Galactic Centre science cases for two E-ELT instrumental projects: MICADO (Davies et al., these proceedings) and EAGLE (Cuby et al., 2008, Rousset et al., these proceedings), as devised by the respective science team (concerning MICADO, see Trippe et al., 2009). Other science cases for EAGLE were also presented during this conference (Evans et al., these proceedings, and Puech et al., these proceedings). We will start by studying gas accretion processes at a couple of parsecs from the black hole, then star formation history in the central parsec, and finally the gravitational potential very close to $\mathrm{Sgr} \mathrm{A}^{*}$.

\subsection{A star formation scenario: the limit cycle hypothesis}

The central region is surrounded by a clumpy ring of molecular gas, the Circumnuclear Disk (CND), with inner radius $2 \mathrm{pc}$ and outer radius $7 \mathrm{pc}$ (Güsten et al., 1987). The stability of this structure is questioned. Authors wonder whether it is permanent or transient. In one scenario, gas piles up in this region until it reaches a critical mass. At this point, it sinks further in, leading to a star formation and accretion event (Morris \& Serabyn, 1996; Morris et al., 1999). It is not clear whether star formation is ongoing in the CND. The hole in the centre of the CND is filled with a complex $\mathrm{H}$ region, Sgr A West or the "Minispiral". It has been demonstrated that its three-arm spiral appearance is a projection effect (Paumard et al., 2004). The Minispiral is a compound of several dust clouds, sheared as they orbit Sgr A*. Their outer layer is ionised. The Northern Arm, the largest and arguably most massive of these clouds, may weigh $\simeq 1000 M_{\odot}$. From its dynamics, it is inferred that it may be one clump of the CND, destabilised from its previous orbit, perhaps due to the interaction with the nearby supernova remnant Sgr A East (Ekers et al., 1975).

This gaseous content is intermixed with the nuclear star cluster. It is mostly composed of a relaxed population of old stars, but is well-known to also harbour more than a hundred "young", massive stars, born in a burst $2-6 \times 10^{6}$ years ago. Their evolutionary state ranges from the main sequence to the most evolved Wolf-Rayet stage. It is agreed that about half of these stars are located within a fairly thin (although of measurable thickness) disk, and have moderate eccentricities (e.g. Genzel et al., 2000; Tanner et al., 2006; Paumard et al., 2006; Lu et al., 2009). The existence of a second, co-eval disk is a matter of debate. All the properties of this disk seem to indicate that in the past, a massive $\left(>10^{4} M_{\odot}\right.$ ), self-gravitating, circularised gaseous disk has occupied the central parsec of the Galaxy. Some of this gas may have been accreted by Sgr A*, but most of it has gone into star formation.

This scenario can be extended a little bit further (Morris et al., 1999): the massive hot stars in the Galactic Centre are responsible for the ionisation of the Minispiral. It is suspected that their feedback plays a major role in the shaping of the CND: the ring seems to be sustained by their collective wind. When these stars eventually die, the equilibrium will be broken. The $\simeq 10^{4} M_{\odot}$ of gas it contains (e.g. Christopher \& Scoville, 2003) will be released in the central cavity. At this point, a new massive disk may form, leading to a new disk of massive stars. As accretion goes on at larger scale, a CND may form again around the nuclear star cluster. Hence, the accretion disk star formation scenario is cyclic, and can lead to successive events of nuclear activity.

With these remarks, it becomes clear that studying star and gas dynamics in the Galactic Centre is complementary, and that both are needed to really understand how a supermassive black holedominated galactic nucleus functions.

It must be added, however, that at this point all the proposed scenarios fail to explain quantitatively a small group of OB stars in the central arcsecond of the Milky-Way, the "S stars". They orbit the black-hole in decades on eccentric orbits and are accelerated to nearly relativistic velocities $(\simeq c / 100)$ at their periapse.

\subsection{MICADO and EAGLE}

In itself, the ELT era will bring direct imaging spatial resolution and sensitivity to unprecedented levels. But an ELT needs to be equipped with correspondingly high-level instrumentation for the benefit to be real. Current wide field astrometric studies in the Galactic Centre are limited by systematic effects, in particular the imperfect knowledge of instrumental distortion of the image. Taking advantage 
of their experience with NAOS/CONICA on the VLT, the MICADO consortium is proposing a camera with large field astrometry as a design driver. Trippe et al. (2009) discuss the various statistical and systematic effects to which MICADO astrometry will be subject and conclude that an accuracy of $40 \mu \mathrm{m}$ is achievable.

The second technique which has brought a harvest of results in the last few years is that of spectroimaging. Integral field spectrometers such as CFHT/BEAR (Maillard, 2000), VLT/SINFONI (Eisenhauer et al. , 2003) and Keck/OSIRIS (Larkin et al., 2003) allow measuring in parallel spectra over a $2 \mathrm{D}$ area, thus permitting the spectral typing of dozens of stars at a time and the measurement of radial velocity maps of gas clouds after suppression of stellar features. Here again, 10-m class telescopes are reaching their limitations as the compromise between spatial and spectral resolution, band width and field of view, is getting more and more difficult to make.

The EAGLE consortium is proposing a multiple integral field unit (multiple IFU) spectrometer similar to KMOS (Sharples et al., 2003). It features $>20$ individual IFUs, positionable in a $5^{\prime}$ patrol field. Each IFU in itself covers $a \simeq 2$ " $\times 2$ " field of view at high spatial resolution (still sampling limited). We will see below how this design can be used efficiently in the case of the Galactic Centre.

\section{The CND-Minispiral interface}

The inner edge of the CND is of particular interest for questioning its stability. Specific measurements include the clump size and mass functions, collisional excitation to get to the cluster wind pressure which applies on the inner edge, 3D velocity of clumps and intra-clump velocity gradients. From these measurements it will be possible to infer the likeliness of clump-clump collisions and to determine how well the orbit of the Northern Arm of the Minispiral matches the kinematics of the CND.

EAGLE is particularly well suited for constructing a radial velocity map over the inner edge of the CND. Ideally, the observer would dream of an integral field spectrometer covering a $1^{\prime} \times 1^{\prime}$ field-ofview at the diffraction limit, and providing high spectral resolution. However such an instrument would be impracticably bulky, complex, expensive, and would deliver a data flow to match these adjectives. The modular design of EAGLE allows distributing the individual IFUs precisely as needed to cover the interesting part of the field. 400 individual IFU fields (2" $\times 2$ ") cover the interface between the CND and the Minispiral. With 20 IFUs, this corresponds to 20 non-redundant pointings. It could presumably be done well within one observing period.

The CND has been studied widely at sub-mm wavelength. Interferometric systems have the disadvantage that they eliminate low spatial frequencies. In addition, the advantage of EAGLE over longer wavelengths is that both the CND and the Minispiral would appear on the same data, the former in molecular lines and the latter in ionised lines. This will ease the positional and velocity registration of the two objects by eliminating systematic effects.

This radial velocity map has to be complemented with sky velocities. MICADO will be ideal for this purpose: it will allow recording the proper motion of gas in the same emission lines as observed with EAGLE.

\section{Nuclear cluster}

To a very large extent, current studies of the nuclear cluster star population are confusion limited. Precision measurements of astrometry and photometry is limited to $K \simeq 16-17.5$, which corresponds to main sequence B stars. MICADO at the E-ELT will push this limit down by $\geq 5$ magnitudes, completing the mass function to sub-solar main sequence stars. The astrometric precision will also be increased by a factor of 3-6. It will be possible to determine orbits of $\simeq 10^{2.5-3}$ individual stars over a decade of observation.

With such a wealth of information it will be possible to establish the current day mass function. In combination with colours and spectra it will then be possible to determine a robust initial mass function (IMF) in the Galactic Center and test the emerging evidence that the IMF in the Galactic Centre region is much flatter than that in the Galactic disk. The star formation history of the nuclear 


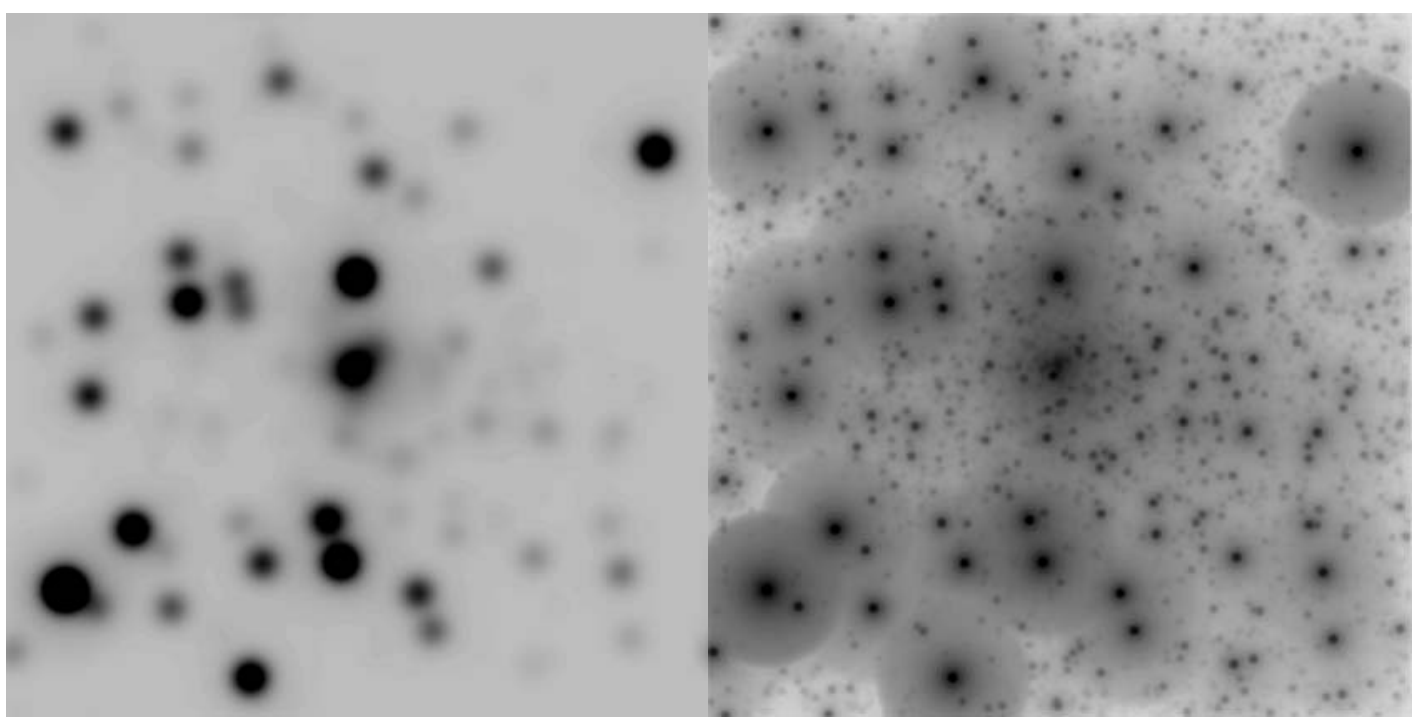

Fig. 1. From Trippe et al. (2009, courtesy of S. Trippe): "An illustration of the E-ELT/MICADO system. These simulated maps show the central 1 " $\times 1$ " (i.e. $8000 \mathrm{AU} \times 8000 \mathrm{AU})$ of the nuclear cluster of the Milky Way at $2.2 \mu \mathrm{m}$. Left panel: The target region as observed with present day $8-10 \mathrm{~m}$ class telescopes. The diffractionlimited resolution is $\simeq 50$ mas. For comparison, with actual observations, see e.g. Genzel et al. (2003); Ghez et al. (2005). Right panel: The same field as seen by MICADO. The angular resolution is $\simeq 10$ mas. The improvement in detail and depth is obvious."

cluster will also become accessible. Has it occurred as a succession of bursts? Are there other older stellar disks in the central parsecs? MICADO on the E-ELT will test the limit cycle hypothesis for star formation (Sect. 1.2).

\section{The Potential around Sgr A*}

The central arcsecond of the Galaxy contains a surprising stellar population. Dozens of OB stars orbit the black hole on relaxed orbits. Those stars cannot have been formed on their current orbit, for the tidal forces prevent star formation that close to Sgr A*. But they are so short-lived and, therefore, young, that it is hard to conceive how they migrated from their nursery to their current location. This double incapacity to explain the existence of these few stars is called the "paradox of youth" (Ghez et al., 2003). The most famous of these "S" stars is S2 or S0-2 (SIMBAD names: "[EG97] S2" and "[GKM98] S0-2"). It has been observed for more than one of its 15.2-year orbits, passing periapse at $1 \%$ of the speed of light.

Those stars are at the limit of the relativistic regime, but within the uncertainties, their orbits remain perfectly Keplerian. There are two ways in which the situation could improve to finally detect relativistic effects in the motion of stars around Sgr A*, such as periapse advance:

- monitoring the already known stars over a longer time baseline, with improved astrometric precision;

- detecting stars in a more relativistic regime.

The S stars will benefit from MICADO's astonishing astrometric capabilities, like the rest of the nuclear cluster. But in addition, MICADO will also allow detecting new stars closer to Sgr A* (Fig. 1). Detection with the current instruments is crowding-limited. With the improved resolution, it will become possible to detect fainter stars, some of which should have tighter, faster orbits around Sgr A* or pass closer during periapse. Relativistic effects will be easier to detect on such stars.

Weinberg et al. (2005) have analysed the tremendous potential of monitoring the motion of stars very close to Sgr A* with ELTs: 
- measuring the distance to the Galactic Centre $\left(R_{0}\right)$ and the mass of Sgr A* to $<0.1 \%$;

- measuring the mass distribution (including dark matter) around the central black hole;

- measuring general relativistic effects;

- detection of stellar remnants through two-body encounters with stars.

\section{Summary}

The Galactic Centre is a unique laboratory for studying the engine at work in galactic nuclei. Investigations with current instrumentation is limited by sensitivity and, above all, spatial resolution. The advent of ELTs will bring quantitative but also qualitative progresses in the understanding of this extreme environment. It will allow measurements and analyses which are out of reach of 10-m telescopes, in several domains:

- Gas accretion mechanisms at parsec scale;

- Star formation history;

- The potential around Sgr A*:

- Extended mass component;

- General relativistic effects;

- Dark matter composition (smooth distribution of particles vs. discrete distribution of stellar remnants).

\section{References}

Christopher, M. H., \& Scoville, N. Z., ASP Conf. Ser. 290, (2003) 389-390

Cuby, J.-G., Morris, S.; Bryson, I., Lehnert, M., Evans, C., Fusco, T., Jagourel, P., Myers, R., Rousset, G., Schnetler, H., Amans, J.-P., Allington-Smith, J, Assemat, F., Beard, S., Chemla, F., Content, R., Dipper, N., Ferrari, M., Gendron, É., Gimenez, J.-L., Hastings, P., Hubert, Z., Hugot, E., Laporte, P., Leroux, B., Madec, F., Neichel, B., Morris, T., Prieto, É., Swinbank, M., Talbot, G., Taylor, W., Vidal, F., Vivès, S., Vola, P., \& Wells, M., Proc. SPIE 7014, (2008) 70141K-1-70141K-11

Eisenhauer, F., Abuter, R., Bickert, K., Biancat-Marchet, F., Bonnet, H., Brynnel, J., Conzelmann, R. D., Delabre, B., Donaldson, R., Farinato, J., Fedrigo, E., Genzel, R., Hubin, N., Iserlohe, C., Kasper, M., Kissler-Patig, M., Monnet, G., Roehrle, C., Schreiber, J., Stroebele, S., Tecza, M., Thatte, N., \& Weisz, H., Proc. SPIE 4841, (2003) 1548-1561

Ekers, R. D., Goss, W. M., \& Schwarz, U., J., A\&A 43, (1975) 159-166

Genzel, R., Pichon, C., Eckart, A., Gerhard, O. E., \& Ott, T., MNRAS 317, (2000) 348-374

Genzel, R., Schödel, R., Ott, T., Eisenhauer, F., Hofmann, R., Lehnert, M., Eckart, A., Alexander, T., Sternberg, A., Lenzen, R., Clénet, Y., Lacombe, F., Rouan, D., Renzini, A., \& Tacconi-Garman, L. E., ApJ 594, (2003) 812-832

Ghez, A. M., Duchêne, G., Matthews, K., Hornstein, S. D., Tanner, A., Larkin, J., Morris, M., Becklin, E. E., Salim, S., Kremenek, T., Thompson, D., Soifer, B. T., Neugebauer, G., \& McLean, I., ApJL 586, (2003) 127-131

Ghez, A. M., Hornstein, S. D., Lu, J. R., Bouchez, A., Le Mignant, D., van Dam, M. A., Wizinowich, P., Matthews, K., Morris, M., Becklin, E. E., Campbell, R. D., Chin, J. C. Y., Hartman, S. K., Johansson, E. M., Lafon, R. E., Stomski, P. J., \& Summers, D. M., ApJ 635, (2003) 1087-1094

Güsten, R., Genzel, R., Wright, M. C. H., Jaffe, D. T., Stutzki, J., \& Harris, A. I., ApJ 318, (1987) $124-138$

Morris, M., \& Serabyn, E., ARA\&A 34, (1996) 645-702

Morris, M., Ghez, A., \& Becklin, E., AdSpR 23, (1999) 959-968

Larkin, J. E., Quirrenbach, A., Krabbe, A., Aliado, T., Barczys, M., Brims, G., Canfield, J., Gasaway, T. M., LaFreniere, D., Magnone, N., Skulason, G., Spencer, M., Sprayberry, D., \& Weiss, J., Proc. SPIE 4841, (2003) 1600-1610

Lu, J. R., Ghez, A. M., Hornstein, S. D., Morris, M. R., Becklin, E. E., \& Matthews, K., ApJ 690, (2009) 1463-1487 
Maillard, J.-P., ASP Conf. Ser. 195, (2000) 185-190

Paumard, T., Maillard, J.-P., \& Morris, M., A\&A 426, (2004) 81-96

Paumard, T., Genzel, R., Martins, F., Nayakshin, S., Beloborodov, A. M., Levin, Y., Trippe, S., Eisenhauer, F., Ott, T., Gillessen, S., Abuter, R., Cuadra, J., Alexander, T., \& Sternberg, A., ApJ 643, (2006) 1011-1035

Sharples, R. M., Bender, R., Hofmann, R., Genzel, R., \& Ivison, R. J., Proc. SPIE 4841, (2003) 15621571

Tanner, A., Figer, D. F., Najarro, F., Kudritzki, R. P., Gilmore, D., Morris, M., Becklin, E. E., McLean, I. S., Gilbert, A. M., Graham, J. R., Larkin, J. E., Levenson, N. A., \& Teplitz, H. I., ApJ 641, (2006) 891-904

Trippe, S., Davies, R., Eisenhauer, F., Förster Schreiber, N. M., Fritz, T. K., \& Genzel, R., MNRAS submitted, (2009)

Weinberg, N. N., Milosavljević, M., \& Ghez, A. M., ASP Conf. Ser. 338, (2005) 252-259 